\title{
Ueber Interpolation nach der Methode der kleinsten Quadrate.
}

\author{
(Von C. W. Borchardt.)
}

Indem ich in einer Abhandlung, welche in den Schriften der hiesigen Academie erscheint, eine Interpolationsformel für eine Art symmetrischer Functionen aufstellte und dieselbe auf verschiedene Probleme der Algebra anwandte, unter anderen auf die Tschebischefsche Aufgabe, eine ganze Function gegebener Ordnung nach der Methode der kleinsten Quadrate zu bestimmen, wenn eine gröfsere Anzahl von Werthen derselben gegeben ist, als sie nach ihrer Ordnung anzunehmen fähig ist, - gelangte ich aufser den von Herrn Tschebischef selbst gegebenen Formen für die Lösung dieser Aufgabe zu einigen anderen, von welchen die eine, die das Ergebnifs in combinatorischer Gestalt liefert, schon um deswillen bemerkenswerth ist, weil sie, ohne irgend eine Rechnung zu erfordern, sich als blofse Folgerung aus der allgemeinen Regel erweist, die Jacobi im $22^{\text {sten }}$ Bande dieses Journals für die Bestimmung nach der Methode der kleinsten Quadrate gegeben hat. Jacobi stellt sich nämlich die Frage, wie sich der Werth, den die Methode der kleinsten Quadrate zur Lösung eines überzähligen *) Systems linearer Gleichungen für eine einzelne Unbekannte liefert, aus allen den Werthen zusammensetzt, die man für dieselbe Unbekannte erhält, wenn man aus dem gegebenen überzähligen System auf alle Arten ein vollzähliges herausgreift. Er findet, dafs wenn man für jedes vollzählige System die Determinante bildet und ihr Quadrat als das Gewicht des aus diesem System hervorgehenden Werths betrachtet, das unter

*) Es ist kaum nöthig besonders zu sagen, dafs ein System linearer Gleichungen (die hier immer als unabhängig von einander vorausgesetzt werden) ein überzäbliges genannt wird, wenn die Anzahl der Gleichungen gröfser ist als die der Unbekannten, so dafs man ihnen nicht allen gleichzeitig genügen kann, dagegen ein vollzähliges, wenn beide Anzahlen gleich sind, so dafs man ihnen und zwar nur auf eine Weise genügen kann. 
dieser Hypothese genommene Mittel *) aus allen Werthen derjenige ist, welchen die Methode der kleinsten Quadrate liefert. Diese Jacobische Regel ist natürlich nicht blofs auf jede einzelne Unbekannte anwendbar, sondern ebensowohl auf jeden aus den Unbekannten linear zusammengesetzten Ausdruck.

Die Tschebischefsche Aufgabe ist ein besonderer Fall der von Jacobi betrachteten allgemeinen, nämlich derjenige, wo das System linearer Gleichungen das folgende ist:

$$
\theta \alpha_{r} \mathfrak{F} \alpha_{r}=\theta \alpha_{r} \boldsymbol{A}_{r}
$$

Hier hat man dem Index $r$ die Werthe $1,2, \ldots n$ zu geben, $\mathfrak{F} x$ ist die unbekannte ganze Function $m^{\text {ten }}$ Grades, wo $m<n-1, A_{r}$ der gegebene Werth, den sie für $x=\alpha_{r}$ annehmen soll und $\theta \alpha_{r}$ das Maafs der Genauigkeit der Gleichung $\mathfrak{F} \alpha_{r}=A_{r}$, so dafs nach Multiplication mit $\theta \alpha_{r}$ diese Gleichung für $r=1,2, \ldots n$ ein System von Gleichungen giebt, die alle gleich genau sind (eine Annahme, die Jacobi bei Aufstellung seiner Regel gemacht hat). Die Unbekannten dieses überzähligen Systems sind die Coefficienten von $\mathfrak{F} x$, und $\mathfrak{F} x$ selbst ist eine lineare Function derselben, also nach der Jacobischen Regel bestimmbar. Wählt man aus dem gegebenen überzähligen System irgend ein vollzähliges, d. h. von $m+1$ Gleichungen aus, z. B. die Gleichungen, welche den Werthen $1,2, \ldots m+1$ des Index $r$ entsprechen, so ist die Determinante aus diesem vollzähligen linearen System bekanntlich

$$
\theta \alpha_{1} \theta \alpha_{2} \ldots \theta \alpha_{m+1} \Delta\left(\alpha_{1}, \alpha_{2}, \ldots \alpha_{m+1}\right),
$$

wo $A$ das alternirende Differenzenproduct der Gröfsen $\alpha_{1}, \alpha_{2}, \ldots \alpha_{m+1}$ bezeichnet. Das Quadrat dieses Ausdrucks ist also das Gewicht des aus dem ausgewählten vollzähligen System hervorgehenden Werthes von $\mathfrak{F} x$, d. h. des Werthes

$$
\boldsymbol{A}_{1} \frac{x-\alpha_{2} \ldots x-\alpha_{m+1}}{\alpha_{1}-\alpha_{2} \ldots \alpha_{1}-\alpha_{m+1}}+\cdots+A_{m+1} \frac{x-\alpha_{1} \ldots \ldots x-\alpha_{m}}{\alpha_{m+1}-\alpha_{1} \ldots \alpha_{m+1}-a_{m}},
$$

nach der Jacobischen Regel ergiebt sich also für $\mathfrak{F} x$ der nach der Methode

*) Sind $g_{1}, g_{2}, g_{3}, \ldots$ die Gewichte der Werthe $u_{1}, u_{2}, u_{3}, \ldots$ von $u$, so ist das nach dieser Hypothese genommene Mittel

$$
=\frac{g_{1} u_{1}+g_{2} u_{2}+g_{3} u_{3}+\cdots}{g_{1}+g_{2}+g_{3}+\cdots} .
$$


272 Borchardt, ̈̈ber Interpolation nach der Methode der kleinsten Quadrate. der kleinsten Quadrate bestimmte Werth in combinatorischer Form:

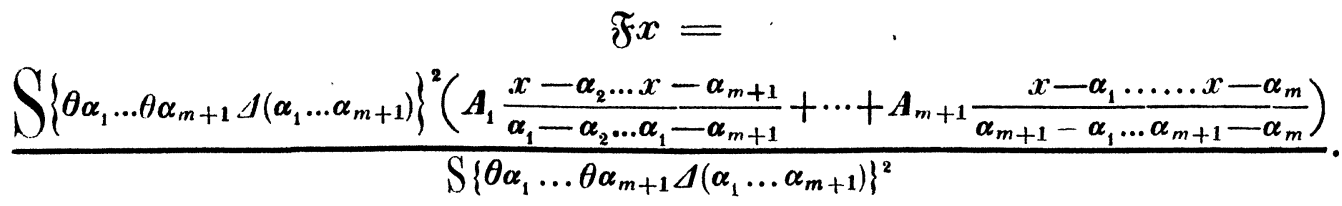
wo die Summen $S$ über alle Combinationen zu $m+1$ der Gröfsen $\alpha_{1}, \alpha_{2}, \ldots \alpha_{n}$ auszudehnen sind.

Dies Resultat ist natürlich nur formell von dem Tschebischefschen verschieden.

Berlin, im September 1860. 